\title{
Effect of Geometry on the Electromagnetic Wave Transport Properties of Photonic Crystals: Comparison of Top-Flat and Top-Curved Refractive Index Profiles
}

\author{
M. Solaimani \\ Qom University of Technology \\ malihe nejati ( $\triangle$ ma.nejati@qom.ac.ir) \\ University of Qom https://orcid.org/0000-0001-8398-9798
}

\section{Research Article}

Keywords: One dimensional plasma photonic crystals, Top-flat and curved refractive index profiles, Transfer matrix method, Geometry effects

Posted Date: October 29th, 2021

DOI: https://doi.org/10.21203/rs.3.rs-1013827/v1

License: (c) (1) This work is licensed under a Creative Commons Attribution 4.0 International License. Read Full License 


\title{
Effect of geometry on the electromagnetic wave transport properties of photonic crystals: comparison of top-flat and top-curved refractive index profiles
}

\author{
M. Solaimani ${ }^{1}$, F. cheraghi ${ }^{2}$, M. Nejati ${ }^{2, a}$ \\ ${ }^{1}$ Department of Physics, Faculty of Mechanical Engineering, Qom University of Technology, Qom 3718146645, Iran \\ ${ }^{2}$ Department of Physics, Faculty of Science, University of Qom, Qom 3716146611, Iran \\ a) Correspondence: ma.nejati@qom.ac.ir
}

\begin{abstract}
In the current paper, we try to engineer the refractive index profile in a one-dimensional photonic crystal as a powerful tool to manage the electromagnetic wave transmission properties. For this purpose, we have compared four sinusoidal, rectangular, triangular, and saw-tooth refractive index profile types. In this way, we have used a transfer matrix method accompanied by the discretization of the spatial domain. This method can readily be applied to any arbitrary continuous refractive index profile. Then, we have tried to address the effects of different geometrical and physical parameters, including the photonic crystal length L, dielectric permittivity $\varepsilon_{d}$, number of layers and plasma density $\mathrm{n}_{\mathrm{p}}$, etc. on the light propagation through the mentioned photonic crystals. In the proposed two-layer plasma/dielectric photonic crystals we could observe acceptable ranges of Omni-directional photonic band gaps that their position width and their number can be regulated. We determine the most and least tunable systems.
\end{abstract}

\section{Keywords}

One dimensional plasma photonic crystals, Top-flat and curved refractive index profiles, Transfer matrix method, Geometry effects

\section{Introduction}

Photonic crystals are defined as artificial structures with a periodic refractive index that can change the electromagnetic wave propagation similar to the periodic potentials in the atomic crystal. They can alter the electron motion by creating an allowed and forbidden electronic spectrum. If the frequency of a propagating wave meets a photonic bandgap, 
electromagnetic wave propagation is prohibited. In other words, the photonic crystal periodicity forbids the propagation of some frequencies throughout the structure. The photonic band gaps essentially depend on the contrast of the dielectric functions of materials in a photonic crystal. They can be interpreted using the interference of the forward and backward traveling waves in the system. Photonic structures have so far been realized in one [1], two [2], and three dimensions [2]. One-dimensional photonic crystals can be fabricated using different techniques, including electrochemical etching, spin coating, and various physical vapor deposition $[3,4]$. The photonic crystals importance arise from their intense applications in optical devices such as optical filters [5], optical switches [6], optical logic devices [7], optical buffers [8], solar cells, 3D fabrication matrices, sensors, color displays fluorescent enhancement devices [9], infrared reflectors [10], etc.

To regulate the physical properties of the devices as mentioned earlier, different concepts and properties of photonic crystals have so far been addressed such as a light-induced color change in the Sapphirinid copepods [11], strong second-harmonic generation [12], defect modes [13], Tamm/Fano resonance [14], the electromagnetic density of modes [15], tunable exciton-polariton [16], phonon-polariton excitations [17], ripple suppression of third-order dispersion [18], optical Contrast Tuning [19], etc.

Also, different effects on the transport properties of photonic crystals are considered, including Pressure, temperature, and thickness [20], applied magnetic fields [21], nonlinearity [22], confined Bloch waves [23], magneto-optical effects [24], etc. an important tool to tune typical properties of the Photonic crystals are their geometries. During these years, different geometries such as rough photonic crystals [25], sinusoidal densities [26], cylindrical photonic crystals [27], etc. as well as different arrangements including, Thue-Morse quasi-photonic crystals [28], Fibonacci [29], Octonacci [30], disordered [31], etc. are investigated. In addition, to have a more profound physical insight, many material types are used in photonic crystals, including plasma [32], magnetized plasma [33], porous silicon [34], metamaterials [35], superconductor-Dielectric [36], Dirac semimetals [37], graphene [38], containing semiconductors [39], Anti-Parity-Time-Symmetric [40], Polymeric [41], mu-negative materials [42], etc.

To consider the transmission properties of a physical system, different methods can be employed. Among these approaches, the transfer matrix has a certain situation. So far this approach has been used in the solution of non-homogeneous anisotropic wave equations [43], 
localization of elastic waves [44], wave propagation in layered anisotropic media [45], prediction of transmission loss of multilayer acoustic materials [46], diffuse-field transmission through multilayered cylinders [47], etc. This method can also be used along with tight-binding models [48], Ising model [49], Monte Carlo schema [50], etc. In addition, the gradient index photonic crystals are considered in some available references such as parabolic refractive index profile [51], hyperbolic-secant refractive index profile [52], saw-tooth refractive index [53], the refractive index of graded layers with linear, exponential, and parabolic profiles [54], and sinusoidal densities [55].

In the current work, we have compared the rectangular, sinusoidal, saw-tooth, and triangular refractive index profiles. A difference between our work and previously studied such as refractive index of graded layers with linear, exponential, and parabolic profiles in [54], is that in their work each layer has a thickness of $\mathrm{L}$, and the refractive index inside this layer changes gradually. However, in our work, we have continuous refractive index profiles thorough the total system spatial domain. Besides in the existing continuous refractive index profiles such as saw-tooth refractive index in ref. [53] or sinusoidal densities of ref. [55], the transmission coefficient has been found using complicated analytical evaluation of the transfer matrix elements. However, in this study, we have used a numerical schema that employs the discretization of the spatial domain. This helps us to study an arbitrary refractive index profile without out much overwhelming effort.

In this research, we consider the electromagnetic wave propagation in plasma photonic crystals containing alternative plasma and dielectric layers. using the transfer matrix method, we have tried to investigate the effects of the plasma layer refractive index, total system length, dielectric constants in the dielectric layers, etc. on the omnidirectional bandgap intervals and the transmission properties of the proposed structures.

\section{Formalism}

The periodicity of the dielectric permittivity of the materials in a typical photonic crystal may lead to the creation of the photonic bandgaps. Here, we produce the dielectric permittivity function using the function $\varepsilon(x)$. These structures have been illustrated in figure (1). 

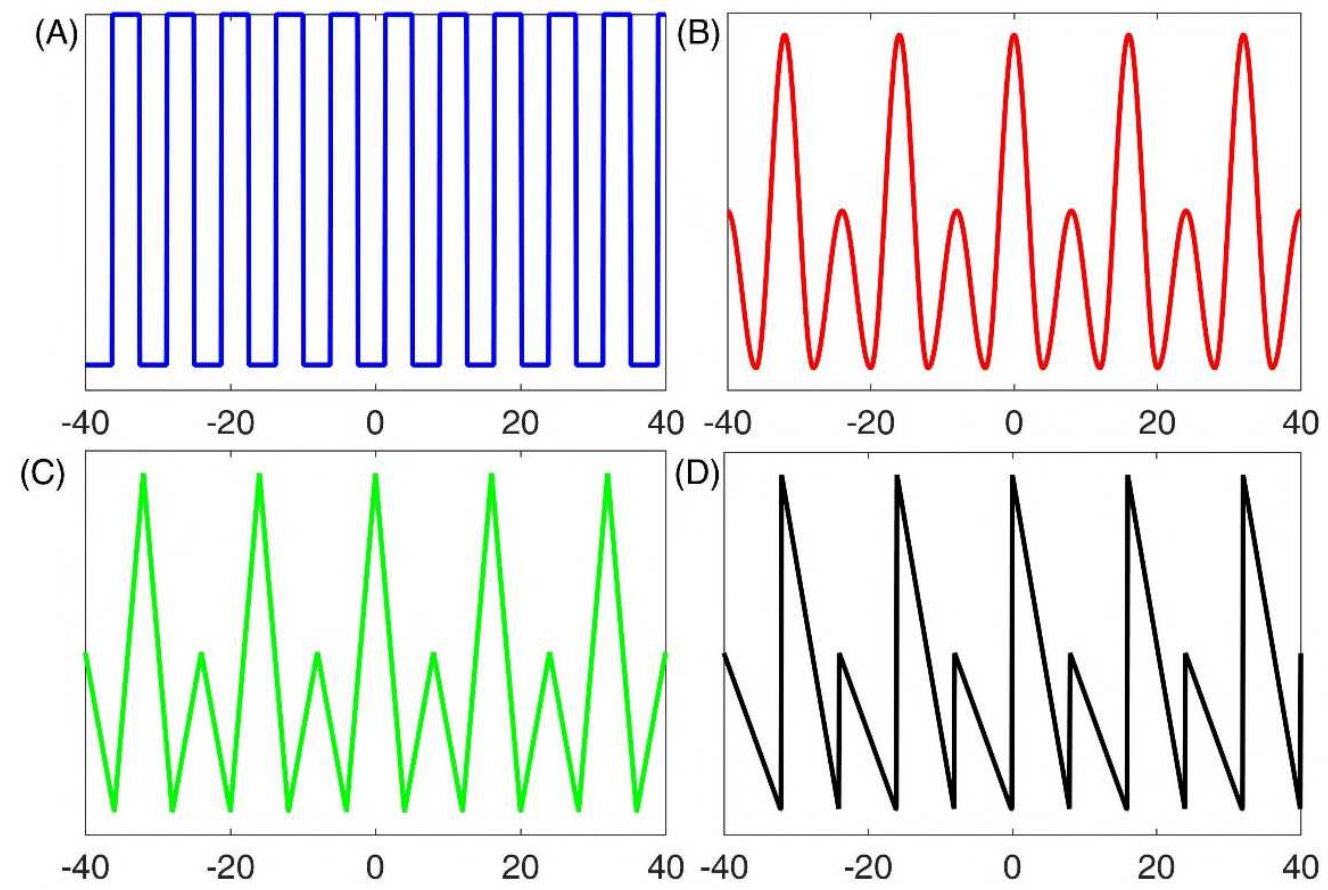

Fig.1 Refractive index profiles of four types of rectangular, sinusoidal, triangular, and saw-tooth photonic crystals in panels (A), to (D), respectively.

We assume some one-dimensional plasma photonic crystals with the rectangular, sinusoidal, triangular, and saw-tooth shapes of layers. We utilize the symbols $n_{p}$ and $n_{d}$ for the refractive index of the plasma and dielectric layers, respectively. The refractive index of the plasma for a non-magnetized plasma is $n_{p}=\left(1-w_{p}^{2} / w^{2}\right)^{1 / 2}$, which the plasma frequency is $w_{p}=\left(n_{e} e^{2} / m e_{0}\right)^{1 / 2}$. Furthermore, $\mathrm{m}$ is the electron mass, $\mathrm{e}$ is the electron charge, and $\mathrm{n}_{\mathrm{e}}$ is the electronic density of the plasma [56]. For transverse electric (TE) and transverse magnetic (TM) waves, the electric field and magnetic fields are perpendicular to the plane of layers. The transfer matrix method (TMM) can connect the electric and magnetic fields at two sides of the neighboring layers using [57],

$M[\Delta z]=\left[\begin{array}{cc}\cos \left(k_{z}^{j} \Delta z\right) & \frac{-i \sin \left(k_{z}^{j} \Delta z\right)}{p_{j}} \\ -i p_{j} \sin \left(k_{z}^{j} \Delta z\right) & \cos \left(k_{z}^{j} \Delta z\right)\end{array}\right]$

Where $k_{z}^{j}=(\omega / c) n_{j} \cos \left(\theta_{j}\right)$ is the $\mathrm{z}$-component of the wave vectors in layer $\mathrm{j}$, and $p_{j}=\sqrt{\varepsilon_{j} / \mu_{j}} \cos \left(\theta_{j}\right)$ where $\left.\cos \theta_{j}=\sqrt{1-\left(n_{0}^{2} \sin ^{2} \theta_{0} / n_{j}^{2}\right.}\right)$. Besides, c, $\theta_{j}, \mathrm{n}_{0}$ and $n_{j}=\sqrt{\varepsilon_{j} \mu_{j}}$ 
are the speed of light in vacuum, the beam angle in layer $\mathrm{j}$, the refractive index of the free space, and the refractive index of the $\mathrm{k}^{\text {th }}$ layer, respectively [58]. Also, $\varepsilon_{\mathrm{i}}$ and $\mu_{\mathrm{i}}$ are the permittivity and the permeability of the materials in $\mathrm{k}^{\text {th }}$ layer, respectively. Now, the total transfer matrix for N-layers reads,

$M^{T o t}=\prod_{j=1}^{N} M_{j}=\left[\begin{array}{ll}M_{11} & M_{12} \\ M_{21} & M_{22}\end{array}\right]$.

The transmission coefficient of the proposed structures can now be written as

$t=\frac{2 p_{0}}{\left(M_{11}+M_{12} p_{s}\right) p_{0}+\left(M_{21}+M_{22} p_{s}\right)}$.

Where we have $p_{0}=n_{0} \cos \left(\theta_{0}\right)$ and $p_{s}=n_{s} \cos \left(\theta_{s}\right)$, in which $\mathrm{n}_{0}, \mathrm{n}_{\mathrm{s}}$ are the refractive index of the environment before the first layer and after the last layer, respectively. Also, $\theta_{0}$ and $\theta_{s}$ are the incident angle and the angle that the light wave leaves the photonic crystal, respectively. The behavior of TM waves can be described using the previous relations with $p_{j}=\sqrt{\mu_{j} / \varepsilon_{j}} \cos \left(\theta_{j}\right), p_{0}=\cos \left(\theta_{0}\right) / n_{0}$ and $p_{s}=\cos \left(\theta_{s}\right) / n_{s}$ [59]. The related transmittance $\mathrm{T}$ for the multilayer is obtained using

$T=\frac{p_{s}}{p_{0}}|t|^{2}$

\section{Results and Discussions}

The transmission coefficient versus the incoming light frequency $\omega$ for rectangular refractive index profile is shown in Fig. 2. In this figure, the effect of different values of the dielectric constant, the system length, plasma electron density, and the number of wells (i.e., the number of valleys in Fig. 1(A) on the transmission spectra have been investigated. To examine the effect of each parameter separately, we fix all the parameters in each section and study only the effect of one parameter. As shown in panel (A) of this figure, as $\varepsilon_{d}$ increases, the frequency gap shifts toward lower frequencies (i.e., redshift). As one can see in panel (B) of Fig. 2, the number of bandgaps increases by increasing the system length. Furthermore, in the system with length $\mathrm{L}=10 \mathrm{~mm}$, at frequencies greater than $10 \mathrm{GHz}$, full transmission is approximately seen. Panel (C) of this figure illustrates that the plasma electron density has little 
effect on the position and bandwidth of the photonic gaps in higher frequency ranges. However, if we consider the frequencies smaller than $20 \mathrm{GHz}$, we see that by increasing the plasma electron density, a photonic bandgap creates and its width grows larger. Therefore, we can readily use different values of the plasma electron densities in optical devices in higher frequency regimes without being worried about changing the photonic gaps with changing plasma electron density. The effect of the number of wells on the transmission is also shown in panel (D) of Fig. 2. As this figure shows, by increasing the well number, the position of the photonic bandgaps shift toward higher frequencies (i.e., blueshift), the number of the bandgaps is reduced and as a result, the bandgap widths increase. Moreover, the panel (D) of this figure illustrates that for wellnumber $=30$, the transmission is nearly perfect in the frequency interval 10-90 GHz.
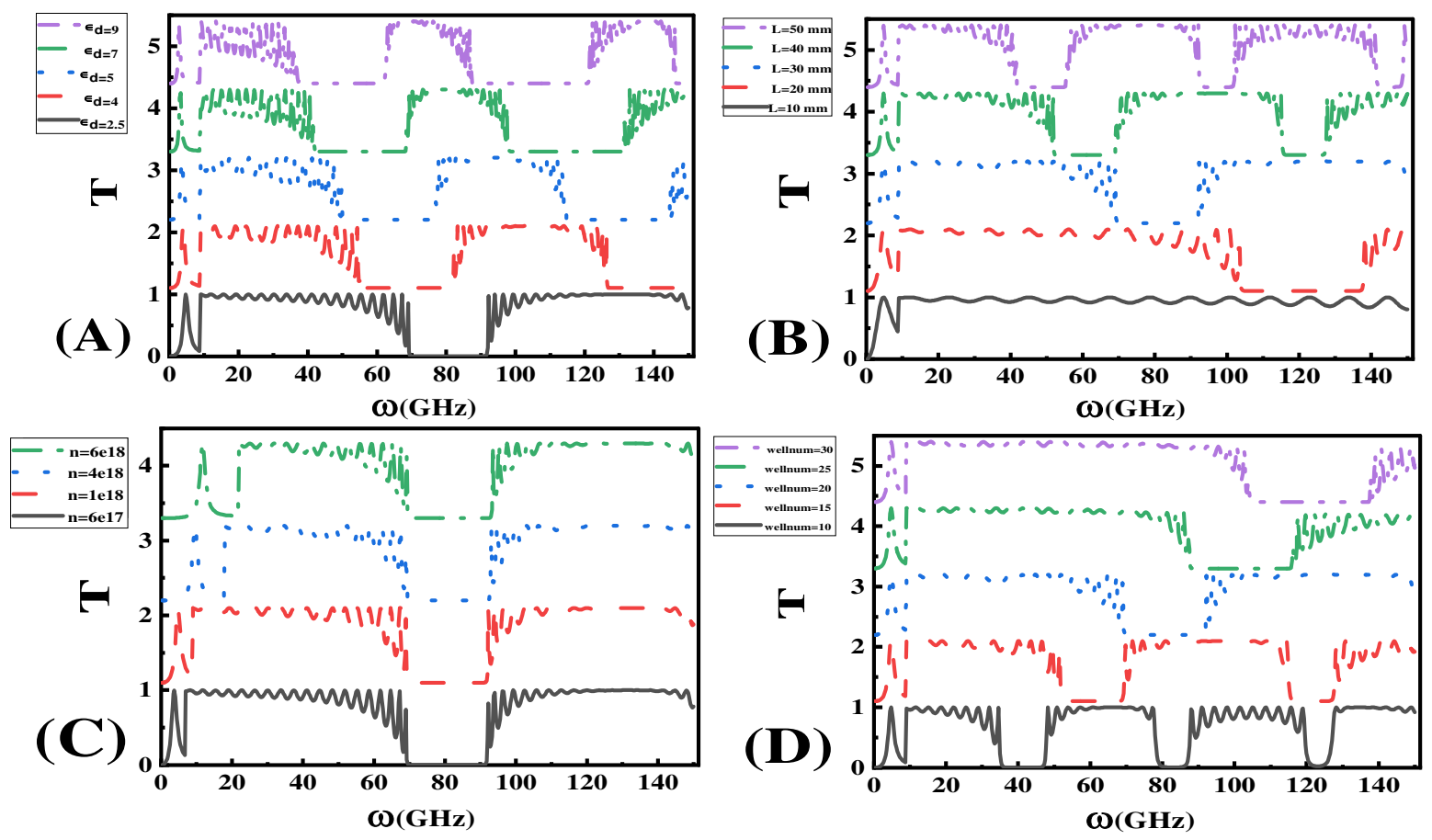

Fig.2 The transmission coefficient versus the incoming light frequency $\omega$ for rectangular refractive index profile. (A) Different values of $\varepsilon_{d}$ have studied the values $L=30 \mathrm{~mm}$, wellnumber $=20, n_{p}=1 \times 10^{18} 1 / \mathrm{m}^{3}$ are utilized. (B) Different values of L are considered. Also, we have used $\varepsilon_{d}=2.5$, wellnumber $=20, n_{p}=1 \times$ $10^{18} 1 / \mathrm{m}^{3}$. (C) Different plasma densities are compared and the values $L=30 \mathrm{~mm}$, wellnumber $=20, \varepsilon_{d}=2.5$, are supposed. (D) Different well numbers are investigated and the values $\varepsilon_{d}=2.5, L=30 \mathrm{~mm}, n_{p}=1 \times 10^{18}$ $1 / m^{3}$ are assumed.

Then, we plot the transmission coefficient versus the incoming light frequency $\omega$ for saw-tooth refractive index profile in Fig. 3. In this figure, the same as Fig. 2, we investigate the effects of different values of dielectric constant, the system length, plasma density, and well number 
on position and the width on the transmission spectra. In panel (A) of Fig. 3, transmission spectra have the redshift when the dielectric constant increased. Comparing panels A of Figs. 2 and 3 reveals that for the same parameters when the refractive index profile has a saw-tooth shape, the number and width of the band gaps are greater than in the rectangular case. In panel (B) of Fig. 3, we investigate the effect of the total system length on the transmission spectra for the saw-tooth refractive index profile. As the length of the system increases, the spectrum redshifts. In panel (B) of Figure 2, for the total system length $L=10 \mathrm{~mm}$ and frequencies greater than $10 \mathrm{GHz}$, the transmission was approximately perfect. However, for the saw-tooth refractive index profile, no transmission is observed for frequencies above $120 \mathrm{GHz}$. Besides, by increasing the system length $\mathrm{L}$, the number of band gaps decreases. Therefore, the widths of the transmission bands and the band gaps decrease by increasing the system length $\mathrm{L}$. The effect of well number on transmission spectra for saw-tooth refractive index profile is investigated in panel (D) of Fig. 3. By increasing the number of wells, the position of band gaps becomes blue shift. In the saw-tooth refractive index profile, the width of transmission bands is reduced compared to the rectangular refractive index profile. Comparing the panels D of figures 2 and 3 also show that, in the systems with rectangular refractive index profile, roughly speaking, the transmission bandwidths are greater than the gap widths, while in the systems with saw-tooth shaped profiles, the gap widths are greater than the transmission bandwidths. thus, according to the optical device requirements, we can use these systems if we want to have greater transmission bands or greater band gaps. In panel (C) of Fig. 3, changing the plasma density has little effect on the position and width of the transmission band. This behavior is similar to the rectangular refractive index profile system. But in the saw-tooth refractive index profile, the bandwidth is almost twice the bandwidth for rectangular refractive index profile. 

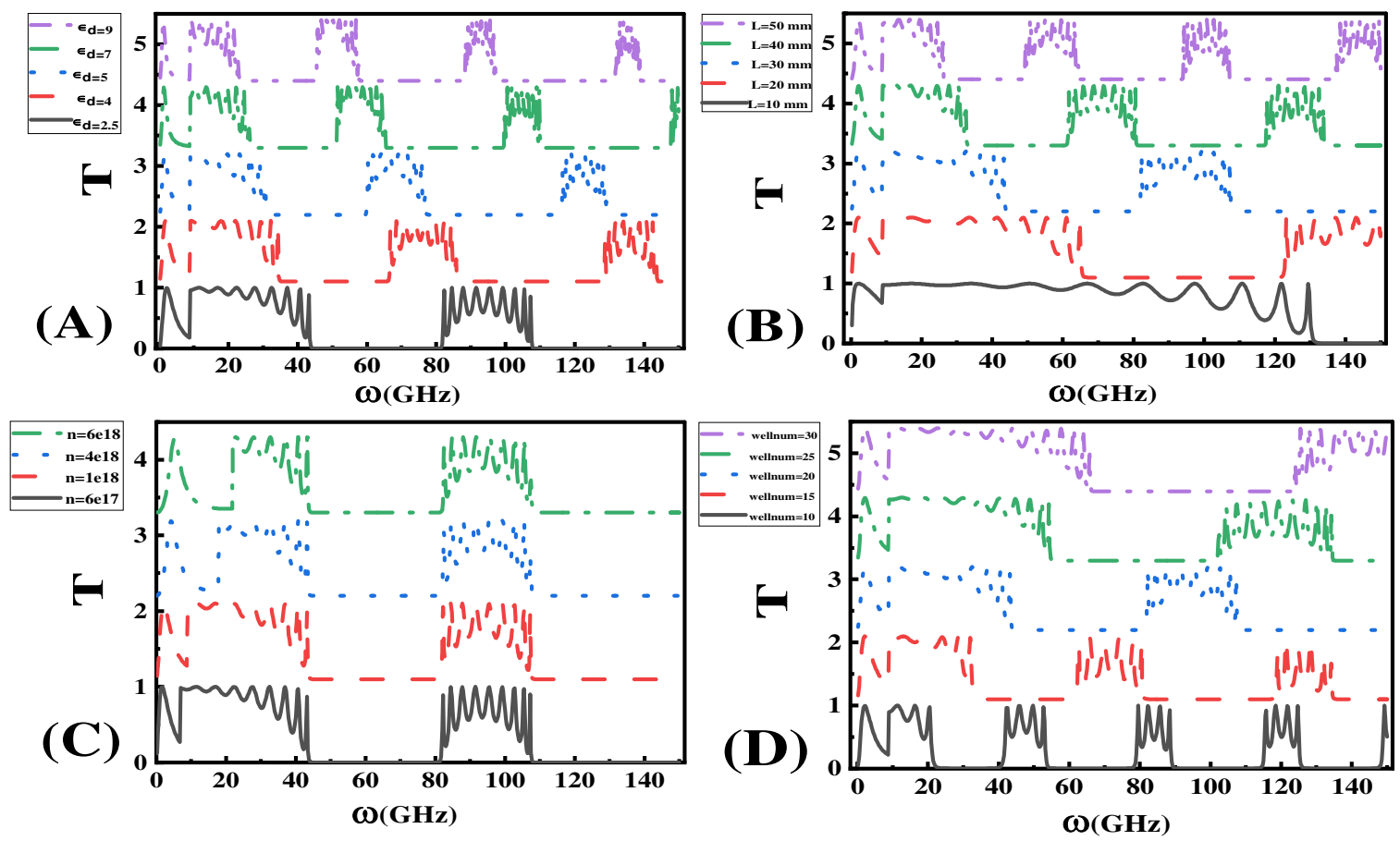

Fig.3 The transmission coefficient versus the incoming light frequency $\omega$ for saw-tooth refractive index profile. (A) Different values of $\varepsilon_{d}$ are used. Also, $L=30 \mathrm{~mm}$, wellnumber $=20, n_{p}=1 \times 10^{18} 1 / \mathrm{m}^{3}$ are considered. (B) Different values of L are studied. Besides, $\varepsilon_{d}=2.5$, wellnumber $=20, n_{p}=1 \times 10^{18} 1 / \mathrm{m}^{3}$ are utilized. (C)

Different plasma densities are compared. Also, $L=30 \mathrm{~mm}$, wellnumber $=20, \varepsilon_{d}=2.5$, are supposed. (D)

Different well numbers are considered while $\varepsilon_{d}=2.5, L=30 \mathrm{~mm}, n_{p}=1 \times 10^{18} 1 / \mathrm{m}^{3}$ are assumed.

To investigate the transmission properties of the plasma photonic crystal with a sinusoidal refractive index profile, we plot Fig. 4. In this figure, the effect of different parameters on the transmission spectra is plotted in panels (A) to (D). In panel (A), $L=$ $30 \mathrm{~mm}$, wellnumber $=20, n_{p}=1 \times 10^{18} 1 / \mathrm{m}^{3}$ are considered and the effect different values of $\varepsilon_{d}$ are investigated. The band gaps positions redshifts as dielectric permittivity $\varepsilon_{d}$ increases. Comparing the panel(A) in this figure with panels (A) of the last two figures shows that the bandgap width for the sinusoidal refractive index profile is greater than the two rectangular and saw-tooth refractive index profiles. In panel (A) of Fig. 4, for the sinusoidal refractive index profile, there is a bandgap in the frequency range of 40 to $45 \mathrm{GHz}$ for all dielectric constants. This case did not exist for rectangular and saw-tooth refractive index profiles. In panel(B), the transmission coefficient versus the incoming light frequency $\omega$ for triangular refractive index profile for different total system lengths is depicted. We see that, by increasing total system length $\mathrm{L}$, the transmission spectra blueshifts. Furthermore, in this panel for $L=10 \mathrm{~mm}$ and the frequency interval $10-70 \mathrm{GHz}$, the transmission is perfect. The panel (C) of Fig. 4 presents the effect of the plasma density on the transmission spectra for the sinusoidal refractive index profile. We observe that the width transmission and bandgap for the sinusoidal profile are 
greater than the two previous profiles. In panel (D) of Fig. 4, we study the effect of well number on the transmission spectra. Here, we changed the number of wells from 10 to 30 and saw that, as the number of wells increases, the number of band gaps decreases, and the width of band gaps increases. So, for wellnumber $=30$, a large bandgap is created in the frequency interval of 62-130 GHz. Considering Figs. 2, 3, and 4, we see that, the behavior of Figs. 2 and 3 are more similar while their characteristics are different from Fig. 2. This can be due to the piecewise shape variation of the refractive index profile in Fig. 2, while Figs. 3 and 4 have topcurved refractive index profiles. Therefore, we expect, the behavior of the triangular shape refractive index profile to be more similar to the Figs. 3 and 4 rather than Fig. 2. Considering Fig. 5 will confirm our claim and as one may see, the overall characteristics of Fig. 5 are similar to Figs. 3 and 4. Investigating panels D of Figs. 4 and 5 also reveals that, there is not a rule of thumb for a variation on the bang gap positions as the number of wells increases. This means, there is not a well-defined increasing or decreasing function for the bang gap positions as the number of wells changes.
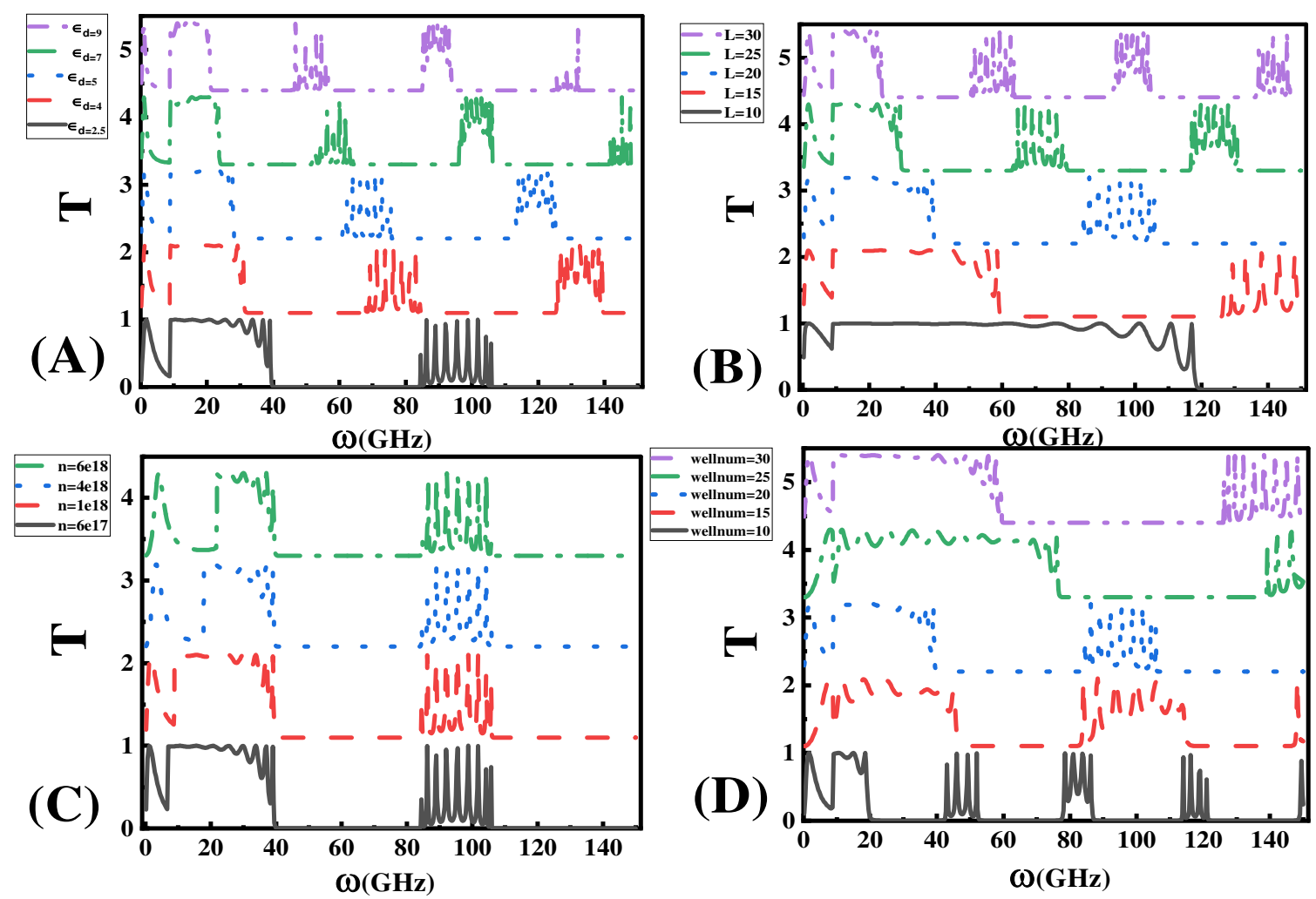

Fig. 4 The transmission coefficient versus the incoming light frequency $\omega$ for sinusoidal refractive index profile.

(A) Different values of $\varepsilon_{d}$ are assumed and $L=30 \mathrm{~mm}$, wellnumber $=20, n_{p}=1 \times 10^{18} 1 / \mathrm{m}^{3}$ are considered.

(B) Different values of $\mathrm{L}$ are compared, while $\varepsilon_{d}=2.5$, wellnumber $=20, n_{p}=1 \times 10^{18} 1 / \mathrm{m}^{3}$ are used. (C)

Different plasma densities are studied and $L=30 \mathrm{~mm}$, wellnumber $=20, \varepsilon_{d}=2.5$, are supposed. (D) Different well numbers are considered and $\varepsilon_{d}=2.5, L=30 \mathrm{~mm}, n_{p}=1 \times 10^{18} 1 / \mathrm{m}^{3}$ are assumed. 

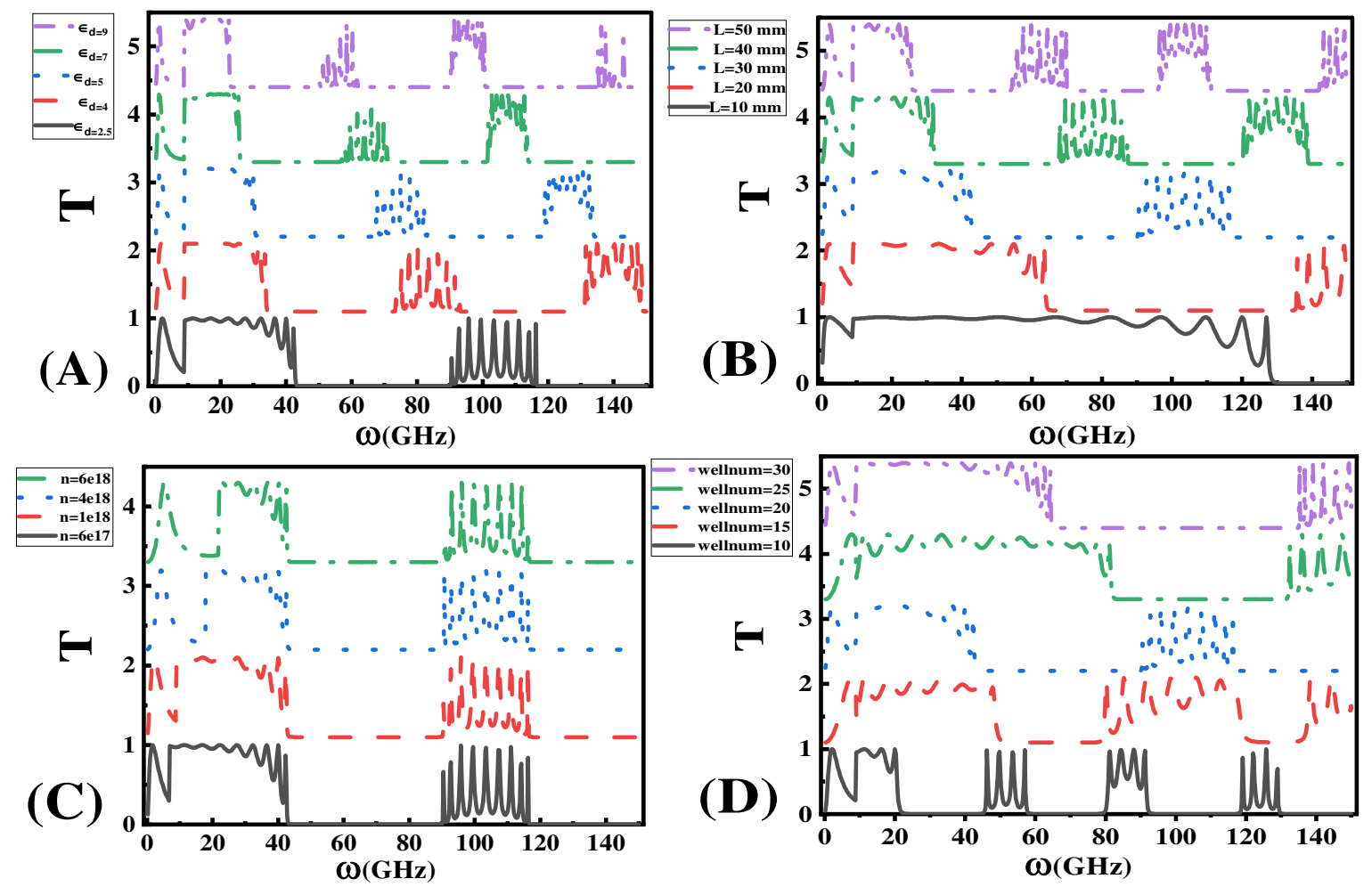

Fig.5 The transmission coefficient versus the incoming light frequency $\omega$ for triangular refractive index profile. (A) Different values of $\varepsilon_{d}$ are studied and $L=30 \mathrm{~mm}$, wellnumber $=20, n_{p}=1 \times 10^{18} 1 / \mathrm{m}^{3}$ are assumed. (B)

Different values of $\mathrm{L}$ are compared, while $\varepsilon_{d}=2.5$, wellnumber $=20, n_{p}=1 \times 10^{18} 1 / \mathrm{m}^{3}$ are used. (C)

Different plasma densities are investigated and $L=30 \mathrm{~mm}$, wellnumber $=20, \varepsilon_{d}=2.5$, are supposed. (D) Different well numbers are considered and $\varepsilon_{d}=2.5, L=30 \mathrm{~mm}, n_{p}=1 \times 10^{18} 1 / \mathrm{m}^{3}$ are assumed.

Since the effect of the plasma electron density was more considerable in the lower frequency regime and had little effect in the higher frequency values, here, we focus ourselves on the lower frequency interval $0-20 \mathrm{GHz}$ and compare four sinusoidal, rectangular, triangular, and saw-tooth refractive index profile types in panels (A) to (D) of Fig. 6, respectively. In this figure, the transmission coefficient versus the incoming light frequency $\omega$ for different plasma densities has been plot. In plotting this figure, we assumed the system length $L=30 \mathrm{~mm}$, dielectric permittivity $\varepsilon_{d}=2.5$, and wellnumber $=20$. In this figure for all four refractive index profile densities, by increasing the plasma density the transmission bands blueshift. Furthermore, In this figure, for all the refractive index profile density per plasma density $n_{p}=$ $6 \times 10^{17} 1 / \mathrm{m}^{3}$, for the frequencies greater than $7 \mathrm{GHz}$, the transmittance is approximately perfect. The rate of the blueshift for rectangular refractive index profiles is faster than other refractive index profiles. 

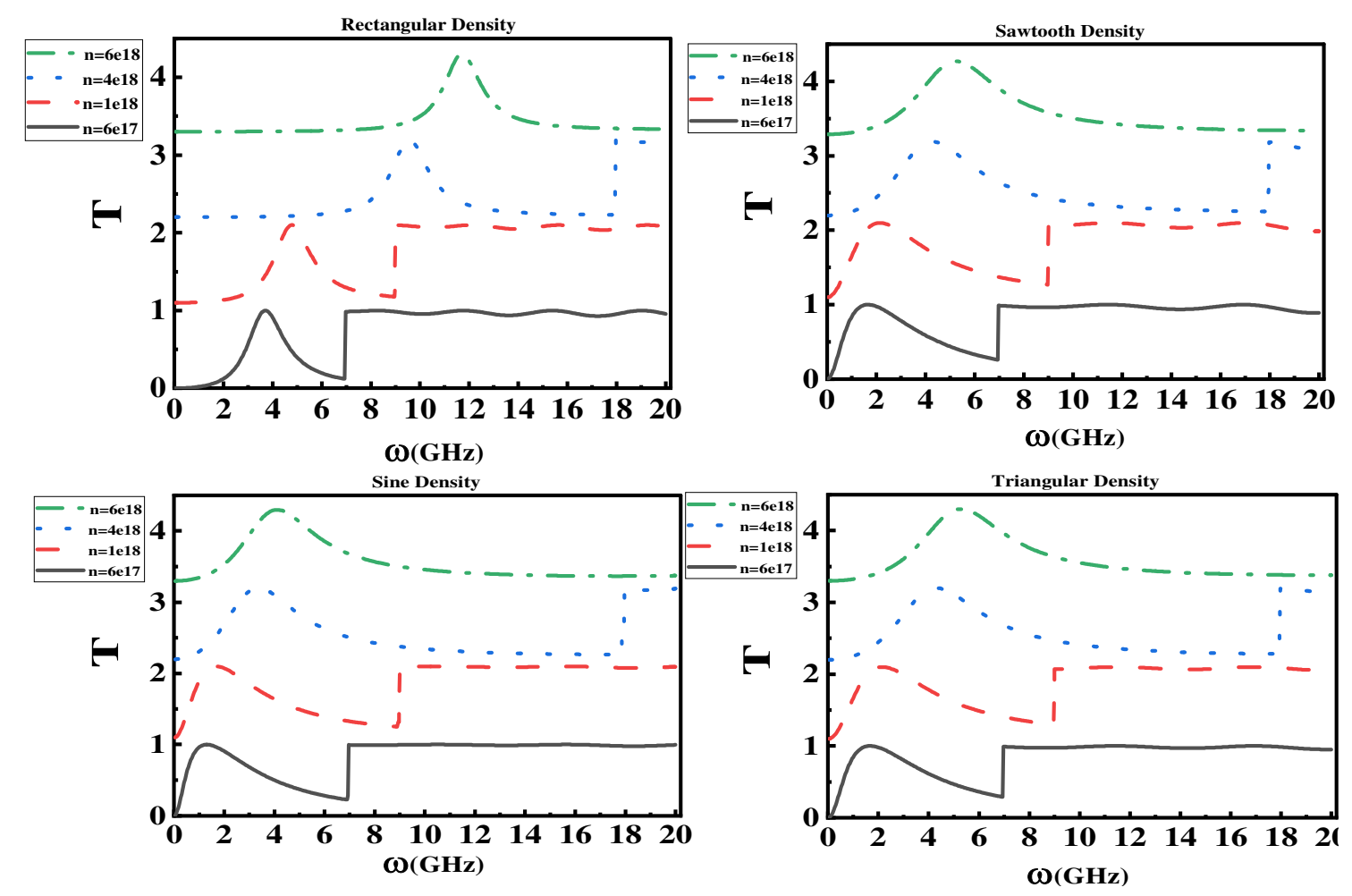

Fig.6 The transmission coefficient versus the incoming light frequency $\omega$ for different plasma densities in four, rectangular, saw- tooth, sinusoidal, and triangular refractive index profiles. (A) Rectangular refractive index profile, (B) Saw-tooth refractive index profile, (C) Sinusoidal refractive index profile, (D) Triangular refractive index profile. The other utilized parameters for all panels are $\varepsilon_{d}=2.5$, wellnumber $=20$, and $\mathrm{L}=30$.

To more clearly see the effect of the refractive index profile type on the transmission spectra, we plot Fig. 7. In this figure, we assume $\varepsilon_{d}=2.5$, wellnumber $=30, n_{p}=6 \times 10^{18}$ $1 / \mathrm{m}^{3}$, and $L=20 \mathrm{~mm}$ and compare different index profiles types. In this figure, there is no transmission in frequencies greater than $100 \mathrm{GHz}$ for saw-tooth, sinusoidal, and triangular refractive index. For rectangular distribution, there is a large transmission band in the frequency interval 20-130 GHz, in which the transmittance is approximately full. Again, we see that the behavior of the rectangular refractive index profile is different from that of the other assumed systems. Besides, the transmission edge around $100 \mathrm{GHz}$ can be tuned by changing the refractive index profile (selecting from saw-tooth, sinusoidal, and triangular ones). 


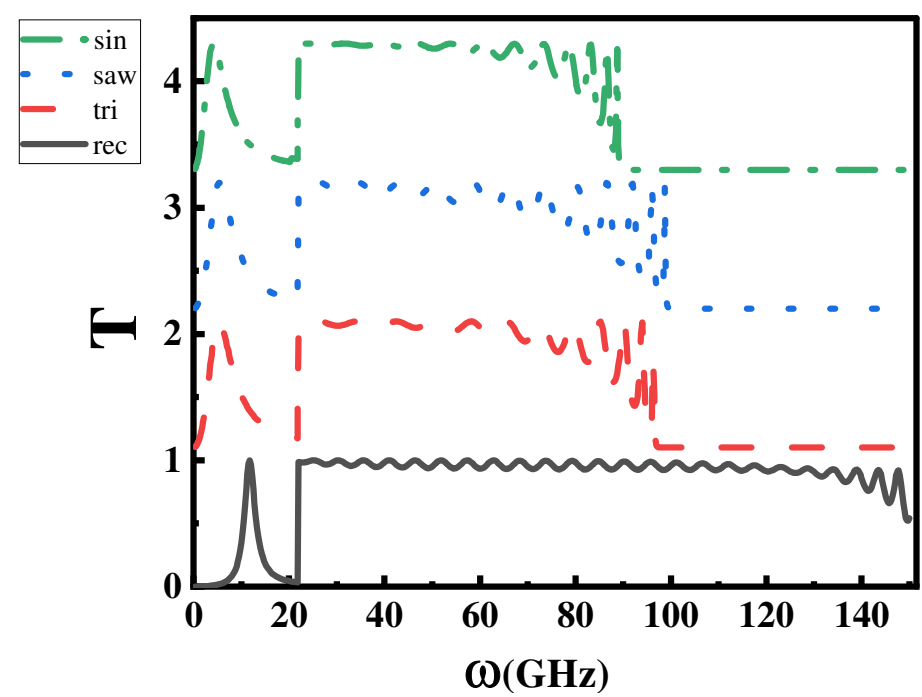

Fig. 7 The transmission coefficient versus the incoming light frequency $\omega$ for four, rectangular, saw-tooth, sinusoidal, and triangular refractive index profile types. The other used parameters for all panels are $\varepsilon_{d}=2.5$, wellnumber $=30, n_{p}=6 \times 10^{18} 1 / \mathrm{m}^{3}$ and $L=20 \mathrm{~mm}$.

Now, for the triangular refractive index profile, we investigate the effect of the incident wave angle on the bandgaps positions and the existence of an omnidirectional bandgap (OBG). We have used total length $\mathrm{L}=30 \mathrm{~mm}$, dielectric permittivity $\varepsilon_{d}=2.5$, well number $=20$ to depict the transmission coefficient versus incident angle, and wave frequency as a contour plot diagram. In panel (A) of Fig. 8, we assume the plasma density equal to $\mathrm{n}_{\mathrm{p}}=6 \mathrm{e} 10^{17} 1 / \mathrm{m}^{3}$, and in panel (B) $6 \mathrm{e} 10^{18} 1 / \mathrm{m}^{3}$. In panel (A) of this figure, in any incident angle in the range between the two solid lines in the range $78<\omega<90 \mathrm{GHz}$, the transmission is forbidden. Therefore, there is an OBG in this area. Panel (B) of Fig. 8 has plotted for a density greater than that used in panel (A). For $\mathrm{n}_{\mathrm{p}}=6 \mathrm{e} 10^{18} 1 / \mathrm{m}^{3}$, there are two gaps in the ranges $16-22 \mathrm{GHz}$ and $78-90 \mathrm{GHz}$. Therefore, in this system, the number of OBGs can be changed using the plasma electron density variation. 

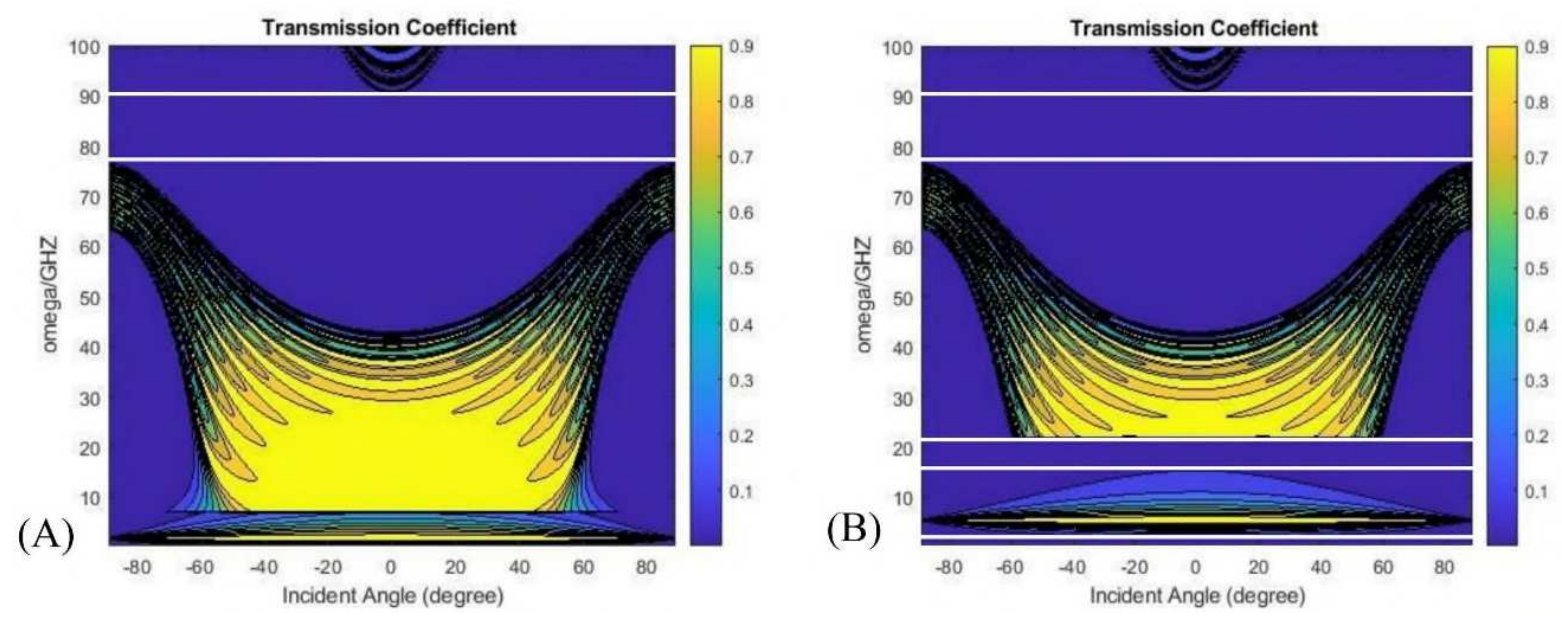

Fig.8 Photonic band structure in terms of incident wave frequency and the incident angle for plasma photonic crystal with triangular refractive index profile. The total system length $\mathrm{L}=30 \mathrm{~mm}$, dielectric permittivity $\varepsilon_{d}=2.5$, well number $=20$ are assumed. In panels $(\mathrm{A})$ and $(\mathrm{B})$, the plasma densities $\mathrm{n}_{\mathrm{p}}=6 \mathrm{e} 10^{17} 1 / \mathrm{m}^{3}$ and $6 \mathrm{e} 10^{18} 1 / \mathrm{m}^{3}$ are respectively utilized.

In Fig. 9, the effect of total system length on the transmission spectra and bandgap position of plasma photonic crystals with triangular refractive index profiles have been investigated. In this figure, well number $=20$, dielectric permittivity $\varepsilon d=2.5$ and plasma density $\mathrm{np}=1 \mathrm{e} 10^{18} 1 / \mathrm{m}^{3}$ are considered. In panel (A), we consider constant total length $\mathrm{L}=10 \mathrm{~mm}$. In panel (A), there is no OBG for this length of the system. In panel (B), of Fig. 8, we have presented $\mathrm{L}=50 \mathrm{~mm}$. In this panel, there are two forbidden bands in the frequency ranges $47<$ $\omega<54 \mathrm{GHz}$ and $94<\omega<96 \mathrm{GHz}$. These bands do not exist in the system with a constant total length $\mathrm{L}=10 \mathrm{~mm}$. Therefore, the system length has a great role in the creation of the OBGs. Comparing this figure with figure 8 reveals that changing the plasma density is more efficient in the creation of OBGs. This is because greater OBGs can be obtained in Fig. 8 and in Fig. 8 the system size can do not grow too large. The later reason (i.e., the fixed system size) may be able to produce optical devices with smaller sizes. 

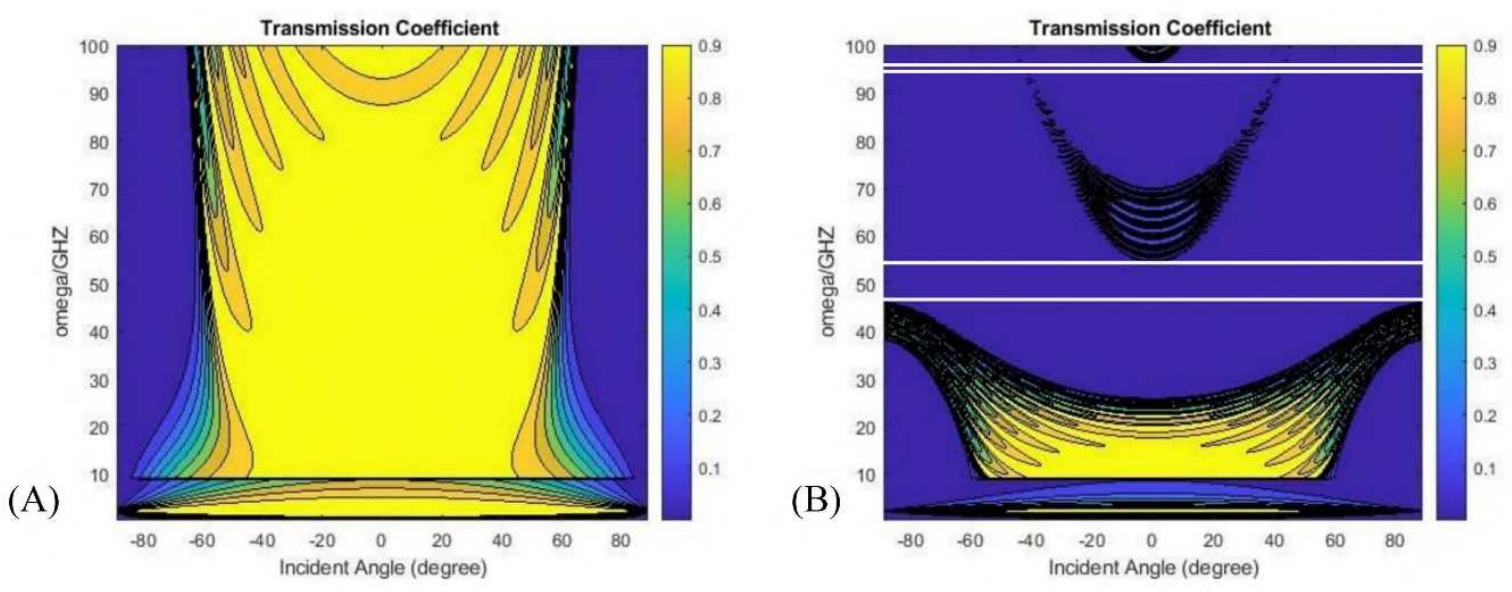

Fig.9 Photonic band structure in terms of incident wave frequency and the incident angle for plasma photonic crystal with triangular refractive index profile. with constant total well number $=20$, dielectric permittivity $\varepsilon_{d}=2.5$, plasma density $\mathrm{n}_{\mathrm{p}}=1 \mathrm{e} 10^{18} 1 / \mathrm{m}^{3}$ are considered. Furthermore, in panels (A) and(B) the system lengths $\mathrm{L}=10 \mathrm{~mm}$ and $\mathrm{L}=50 \mathrm{~mm}$ are respectively utilized.

The effect of dielectric permittivity $\varepsilon_{d}$ on the OBG for the plasma photonic crystal with a triangular refractive index profile is presented in Fig 10. We have used total system length $\mathrm{L}=30 \mathrm{~mm}$, well number $=20$, plasma density $\mathrm{np}=1 \mathrm{e} 10^{18} \mathrm{~m}^{-3}$, and $\varepsilon_{d}=2.5$. In panel (A) of Fig.10, the OBG width is $12 \mathrm{GHz}$. Comparing panel (A) in this figure with panel (B) of figure 9 shows that as the total length of the system increases, the OBG position considerably redshifts. In panel (B) of Fig. 10, we consider $\varepsilon_{d}=9$. In this figure, as the dielectric permittivity increases, the number, and width of the OBGs increase. In this panel, there are three OBGs in the ranges 5-9 GHz, 27-50 GHz, and 74-90 GHz. Comparing the Figs. 8, 9, and 10 also show that the parameter $\varepsilon_{d}$ is more efficient in tuning the OBG width and also regulating the number of OBGs than the plasma electron density and the total system length. This is because wider OBGs and also more OBGs can be achieved by changing the $\varepsilon_{d}$.

(A)

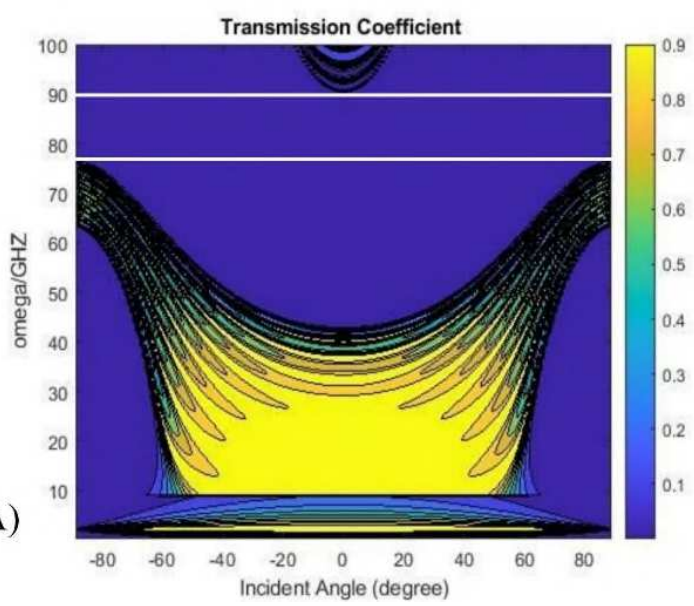

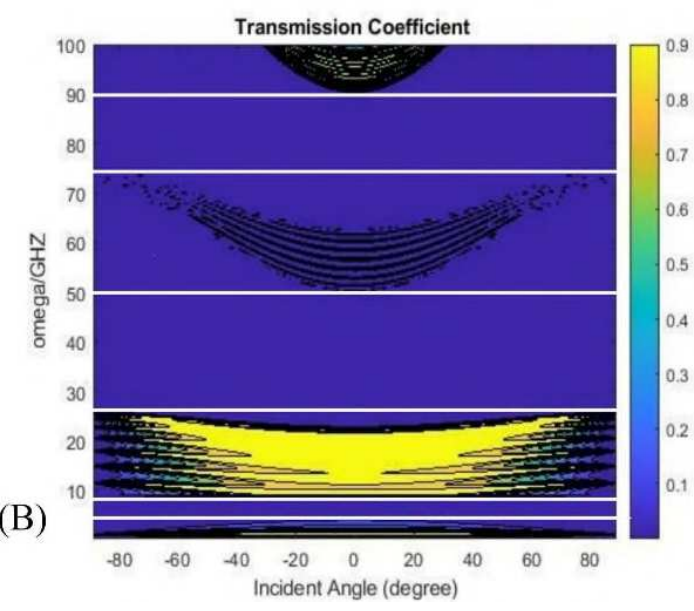


Fig.10 Photonic band structure in terms of incident wave frequency and the incident angle for plasma photonic crystal with triangular refractive index profile. The total system length $\mathrm{L}=30 \mathrm{~mm}$, well number $=20$, plasma density $\mathrm{n}_{\mathrm{p}}=1 \mathrm{e} 10^{18} \mathrm{~m}-3$ are used. Furthermore, in panels (A) and (B), the dielectric permittivities $\varepsilon_{d}=2.5$ and 9 are respectively used.

Finally, the transmission coefficient versus the incoming light frequency and the incident angle for plasma photonic crystal with triangular refractive index profile for both TE and TM polarization modes is shown in Fig. 11. Here, the total system length $\mathrm{L}=30 \mathrm{~mm}$, dielectric permittivity $\varepsilon_{d}=2.5$, plasma density $\mathrm{n}_{\mathrm{p}}=1 \mathrm{e} 10^{18} \mathrm{~m}^{-3}$ are utilized. In panels (A) and (B) of this figure, we consider well numbers 10 and 20, respectively. In panel (A), for well number $=10$, the total OBGs are in the interval between the two solid lines is in the range $39<$ $\omega<45 \mathrm{GHz}$ and $78<\omega<80$. For well number=20 in panel (B), the number of OBG is decreased but the width of OBG is increased. The width of OBG, in this case, is $13 \mathrm{GHz}$. Comparing the Figs. 8, 9, 10, and 11, reveal that the number of OBGs increases by increasing the dielectric constant, system length and the plasma electron density while decreases by increasing the number of wells. Also, we can conclude that the most and the least critical tools to tune the OBGs are the dielectric constant and the total system length, respectively.
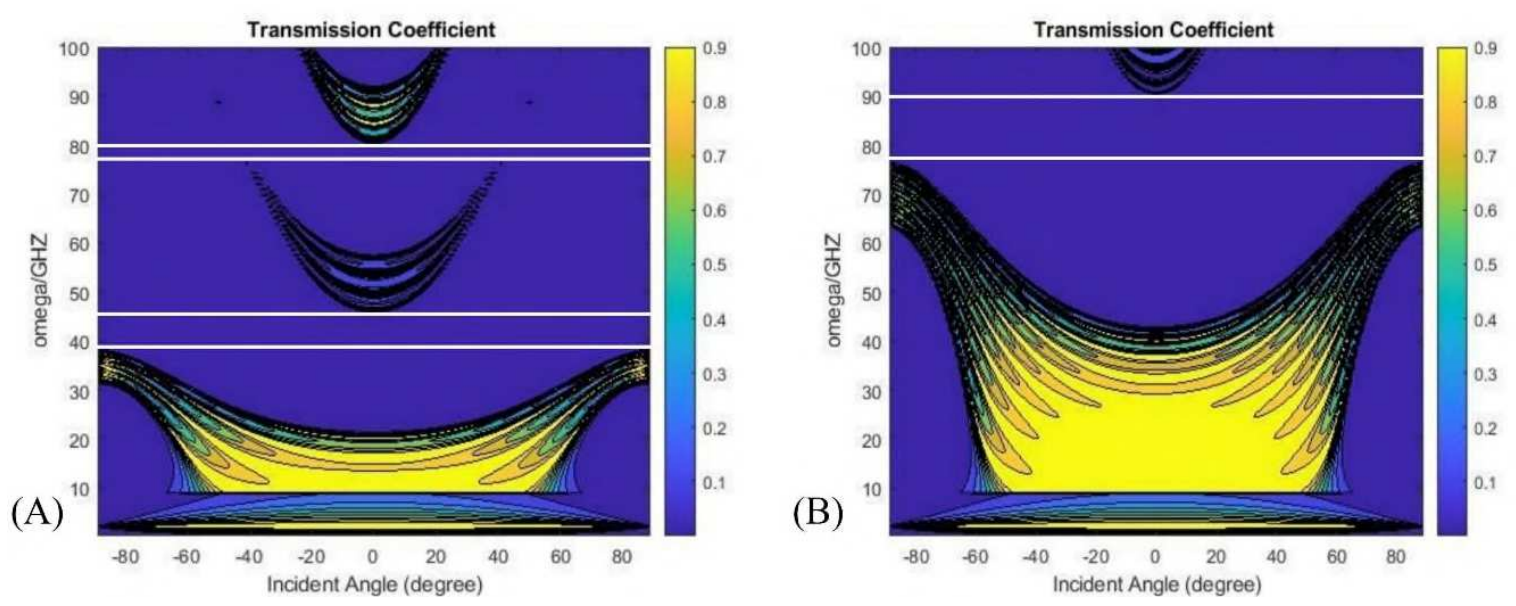

Fig. 11 Photonic band structure in terms of incident wave frequency and the incident angle for plasma photonic crystal with triangular refractive index profile. The total system length $\mathrm{L}=30 \mathrm{~mm}$, dielectric permittivity $\varepsilon d$ $=2.5$, plasma density $n_{p}=1 \mathrm{e}^{18} \mathrm{~m}^{-3}$ are used. Furthermore, in panels $(\mathrm{A})$ and $(\mathrm{B})$, the well numbers 10 and 20 are respectively assumed.

\section{Conclusion}

In this work, we compared the transmission properties of plasma photonic crystals with top-flat rectangular and top-curved sinusoidal, triangular, and saw-tooth refractive index 
profile types. We showed that, for the rectangular refractive index profile, as $\varepsilon_{d}$ increases, the frequency gaps redshift. the number of bandgaps increased by increasing the system length. The plasma electron density had little effect on the position and bandwidth of the photonic gaps in higher frequency ranges. Therefore, one could easily use different plasma electron densities in his/her optical devices in a higher frequency regime without being worried about changing the photonic gaps when plasma electron density changes. Also, by increasing the well number, the position of the photonic bandgaps is blueshifted. For the saw-tooth refractive index profile, the widths of the transmission bands and the band gaps decreased by increasing the system length L. The transmission bandwidths were greater than the gap widths, while in the systems with saw-tooth-shaped profiles the gap widths were greater than the transmission bandwidths. We also concluded that the behavior of the top-curved refractive index profiles is similar. However, they are different from that of top-flat refractive index profiles. Finally, the most and the least critical tools to tune the OBGs were the dielectric constant and the total system length, respectively.

\section{References}

[1] M. Solaimani, M. Ghalandari, A. Aghajamali, "Bandgap engineering in constant total length non magnetized plasma-dielectric multilayers", Optik 207, 164476 (2020).

[2] M. H. Kim, S. H. I'm, O. O. Park, "Rapid Fabrication of Two- and Three-Dimensional Colloidal Crystal Films via Confined Convective Assembly", Adv. Funct. Mater. 15, 1329-1335 (2005).

[3] L. D. Bonifacio, B. V. Lotsch, D. P. Puzzo, F. Scotognella, G. A. Ozin, "Stacking the Nanochemistry Deck: Structural and Compositional Diversity in One-Dimensional Photonic Crystals", Adv. Mater. 21, 1641-1646 (2009).

[4] S. Valligatla, A. Chiasera, S. Varas, N. Bazzanella, D. N. Rao, G. C. Righini, M. Ferrari, "High-Quality Factor 1-D Er3-Activated Dielectric Microcavity Fabricated by RF-Sputtering", Opt. Express 20, 21214-21222 (2012).

[5] Y. Kanamori, N. Matsuyama, K. Hane, "Resonant-wavelength tuning of a pitch-variable 1-D photonic crystal filter at telecom frequencies", IEEE Photon. Technol. Lett. 20, 1136-1138 (2008).

[6] V. Y. Zyryanov, V. A. Gunyakov, S. A. Myslivets, V. G. Arkhipkin, V. F. Shabanov, "Electro-optical switching in a one-dimensional photonic crystal", Mol. Cryst. Liq. Cryst. 488, 1563-5287 (2008).

[7] P. Andalib, N. Granpayeh, "All-optical ultra-compact photonic crystal and gate based on nonlinear ring resonators", J. Opt. Soc. Am. B 26, 10-16 (2009).

[8] T., Baba, "Slow light in photonic crystals", Nat. Photonics 2, 465-473 (2008). 
[9] H. Shen, Z. Wang, Y. Wu, and B. Yang, "One-dimensional photonic crystals: fabrication, responsiveness and emerging applications in 3D construction", RSC Adv. 6, 4505 (2016). [10] K. M. Abohassan, H. S. Ashour, M. M. Abadla, "One-dimensional ZnSe/ZnS/BK7 ternary planar photonic crystals as wide angle infrared reflectors", Results in Physics 22, 103882 (2021).

[11] D. Gur, B. Leshem, V. Farstey, D. Oron, L. Addadi, and S. Weiner, "Light-Induced Color Change in the Sapphirinid Copepods: Tunable Photonic Crystals" Adv. Funct. Mater. 26, 1393-1399 (2016).

[12] S. Zhang, X. Zhang, "Strong second-harmonic generation from bilayer-graphene embedded in onedimensional photonic crystals", Journal of the Optical Society of America B 33, 452-460 (2016).

[13] T.-C. King, C.-J. Wu, "Properties of defect modes in one-dimensional symmetric defective photonic crystals ", Physica E 69, 39-46 (2015).

[14] A. Mehaney, M. M. Abadla, H. A. Elsayed, "1D porous silicon photonic crystals comprising Tamm/Fano resonance as high performing optical sensors", Journal of Molecular Liquids 322, 114978 (2021).

[15] A. Aman, S. Prasad, S. Prakash, G. Sharma, V. Singh, "Controlling the band structures and electromagnetic density of modes in one-dimensional photonic crystals with Lamb wave", Waves in Random and Complex Media, (2020), DOI: 10.1080/17455030.2020.1810362.

[16] E. S. Sedov, E. D. Cherotchenko, S. M. Arakelian, and A. V. Kavokin, "Light propagation in tunable excitonpolariton one-dimensional photonic crystals", Phys. Rev. B 94, 125309 (2016).

[17] K. Casey Huang, P. Bienstman, J. D. Joannopoulos, and K. A. Nelson, S. Fan, "Phonon-polariton excitations in photonic crystals", Phys. Rev. B 68, 075209 (2003).

[18] R. Shiri, H. Shahrokhabadi, T. F. Khalkhali \& A. Bananej, "Ripple suppression of third-order dispersion by using graded index one-dimensional photonic crystals", Journal of Modern Optics, 66, 1045-1051 (2019).

[19] V. A. Tolmachev, A. V. Baldycheva, S. A. Dyakov, K. Berwick, and T. S. Perova, "Optical Contrast Tuning in Three-Components One-Dimensional Photonic Crystals", J. Lightwave Technol. 28, 1521 (2010).

[20] A. Y. Herrera, J. M. Calero, and N. Porras-Montenegro, "Pressure, temperature, and thickness dependence of transmittance in a 1D superconductor-semiconductor photonic crystal", Journal of Applied Physics 123, 033101 (2018)

[21] M S Vasconcelos and M G Cottam, "Theory for polaritons in graphene photonic crystals in an applied magnetic field", J. Phys. D: Appl. Phys. 53, 135101 (2020).

[22] L.-M. Zhao, B.-Y. Gu, Y.-S. Zhou, "Away for enhancing second harmonic generation in one-dimensional nonlinear photonic crystals", Optics Communications 281, 2954-2958 (2008).

[23] A. Pryamikov, K. Joulain, P. Ben-Abdallah, J. Drevillon, "Role of confined Bloch waves in the near field heat transfer between two photonic crystals", Journal of Quantitative Spectroscopy \& Radiative Transfer 112, 1314-1322 (2011). 
[24] S Shukla, S. Prasad, and V. Singh, "Investigation of magneto-optical effects on properties of surface modes in one dimensional magnetized plasma photonic crystals", Phys. Plasmas 23, 092111 (2016).

[25] L. L. Missoni, G. P. Ortiz, M. Luz Martínez Ricci, V. J. Toranzos, W. Luis Mochan, " Rough 1D photonic crystals: A transfer matrix approach ", Optical Materials 109 (2020) 110012.

[26] L. Qi, L. Shang, and S. Zhang, "One-dimensional plasma photonic crystals with sinusoidal densities", Physics of Plasmas 21, 013501 (2014).

[27] Q.-Y. Wang, S. Liu, D. Gui and H.-F. Zhang, "Nonreciprocal absorption characteristics of one-dimensional cylindrical magnetized plasma photonic crystals", Phys. Scr. 96, 065501 (2021).

[28] M. Solaimani, M. Ghalandari, and Malihe Nejati, "Optical filters based on fixed length Thue-Morse plasmadielectric photonic band multilayers: Comparing two, three, and four materials systems", AIP Adv. 11, 025309 (2021).

[29] F. Asadollah Zarif, M. Khazaei Nezhad, H. Rastegrar Moghaddam Rezaieun, "Enhancement of efficiency of second-harmonic generation from MoS2 monolayers in 1D Fibonacci photonic crystals", Photonics and Nanostructures - Fundamentals and Applications 36, 100726 (2019).

[30] E.R. Brandao, C. H. Costa, M. S. Vasconcelos, D. H. A. L. Anselmo, V. D. Mello, "Octonacci photonic quasicrystals", Optical Materials 46, 378-383 (2015).

[31] J.-H. Wu, M. Artoni, and G. C. La Rocca, "Perfect absorption and no reflection in disordered photonic crystals", Phys. Rev. A 95, 053862 (2017).

[32] H. Mehdian, Z. Mohammadzahery, and A. Hasanbeigi, "Analysis of plasma-magnetic photonic crystal with a tunable bandgap", Physics of Plasmas (1994-present) 20, 043505 (2013).

[33] Y. Ma, H. Zhang and C. Hu, "Tunable omnidirectional bandgap and polarization splitting in one-dimensional magnetized plasma photonic crystals with a quasi-periodic topological structure", J. Opt. 22, 025101 (2020).

[34] C. Felipe Ramirez-Gutierrez, H. David Martinez-Hernandez, I. Alonso Lujan-Cabrera \& M. E. nrique Rodriguez-García, "Design, fabrication, and optical characterization of one dimensional photonic crystals based on porous silicon assisted by in-situ photoacoustics", Scientific Reports 9, 14732 (2019).

[35] A. A. Shokri and R. Jamshidi, "Merging of defect modes in a superlattice of one-dimensional metamaterials photonic crystals", AIP Advances 9, 055318 (2019).

[36] H.-M. Lee and J.-C. Wu, "Transmittance spectra in one-dimensional superconductor-dielectric photonic crystal", J. Appl. Phys. 107, 09E149 (2010).

[37] Y. Zhao, Y. Zhang, X. Guo, M. Liu, H. Chen, S. Liu, and H. Zhang, "Tunable omnidirectional photonic bandgap of one-dimensional photonic crystals containing Dirac semimetals", J. Appl. Phys. 122, 223108 (2017).

[38] L. Bian, P. Liu, G. Li, Z. Lu, C. Liu, "Characterization for one-dimensional graphene embedded photonic crystals at terahertz frequencies", Opt Quant Electron 48, 436 (2016). 
[39] X. Dai, Y. Xiang, S. Wen, and H. He, "Thermally tunable and omnidirectional terahertz photonic bandgap in the one-dimensional photonic crystals containing semiconductor InSb", J. Appl. Phys. 109, 053104 (2011).

[40] H. Wang, W. Kong, P. Zhang, Z. Li, and D. Zhong, "Coherent Perfect Absorption Laser Points in OneDimensional Anti-Parity-Time-Symmetric Photonic Crystals", Appl. Sci. 9, 2738 (2019).

[41] P. Lova, H. Megahd, P. Stagnaro, M. Alloisio, M. Patrini, and D. Comoretto, "Strategies for Dielectric Contrast Enhancement in 1D Planar Polymeric Photonic Crystals", Appl. Sci. 10, 4122 (2020).

[42] Y. Liu, L. Yi, X.-G. Hu, Y.-F. Duan, and Z.-Z. Yang, "Phase retarder based on one-dimensional photonic crystals composed of plasma and mu-negative materials", Physics of Plasmas 22, 012101 (2015).

[43] K. Mehrany and S. Khorasanim "Analytical solution of non-homogeneous anisotropic wave equations based on differential transfer matrices", J. Opt. A: Pure Appl. Opt. 4 (2002).

[44] R. Sepehrinia, M. R. Rahimi Tabar, and M. Sahimi, "Numerical simulation of the localization of elastic waves in two- and three-dimensional heterogeneous media", Phys. Rev. B 78, 024207 (2008).

[45] L. Wang, and S. I. Rokhlin, "Stable reformulation of transfer matrix method for wave propagation in layered anisotropic media", Ultrasonics, 39, 413-424 (2001).

[46] C. M. Lee, and Y. Xu, "A modified transfer matrix method for prediction of transmission loss of multilayer acoustic materials", Journal of Sound and Vibration, 326, 290-301 (2009).

[47] A. Parrinello, K. Kesour, G. L. Ghiringhelli, and N. Atalla, "Diffuse field transmission through multilayered cylinders using a Transfer Matrix Method", Mechanical Systems and Signal Processing, 136, 106514 (2020).

[48] T. Kostyrko, "Transfer-matrix approach for modulated structures with defects", Phys. Rev. B 62, 2458-2465 (2000).

[49] D. C. Mattis and P. Paul, "Statistical Transfer Matrix of a Spin-Glass Ladder", Phys. Rev. Lett. 83, 37333736 (1999).

[50] H. Saleur and B. Derrida, "A combination of Monte Carlo and transfer matrix methods to study 2D and 3D percolation", J. Physique 46, 1043-1057 (1985).

[51] R. Shiri, A. Bananej, and Safari, E., 2016. Compression of ultra-short light pulses using the graded refractive index one-dimensional photonic crystals. Optics Communications, 375, pp.23-28.

[52] D. Yilmaz, I. H. Giden, M. Turduev, and H. Kurt, "Design of a Wavelength selective medium by graded index photonic crystals", IEEE Journal of Quantum Electronics, 49, 477-484 (2013).

[53] G. V. Morozov, D. W. L. Sprung, and J. Martorell, "One-dimensional photonic crystals with a sawtooth refractive index: another exactly solvable potential", New Journal of Physics, 15, 103009 (2013).

[54] R. Shiri, A.Bananej, E.Safari, "Compression of ultrashort light pulses using the graded refractive index onedimensional photonic crystals", Optics Communications 375, 23-28 (2016). 
[55] L. Qi, L. Shang, and S. Zhang, "One-dimensional plasma photonic crystals with sinusoidal densities", Physics of Plasmas 21, 013501 (2014).

[56] Z. Naderi Dehnavi, H. Ranjbar Askari, M. Malekshahi, and D. Dorranian, "Investigation of tunable omnidirectional bandgap in 1D magnetized full plasma photonic crystals", Physics of Plasmas 24, 093517 (2017), [57] A. Aghajamali, M. Barati, "Effects of normal and oblique incidence on zero-n gap in periodic lossy multilayer containing double-negative materials”, Physica B 407, 1287-1291 (2012).

[58] L. Zhang, Y. Zhang, L. He, Z. Wang, H. Li and H. Chen, "Zero- n gaps of photonic crystals consisting of positive and negative index materials in microstrip transmission lines", J. Phys. D. Appl. Phys. 40, 2579-2583 (2007).

[59] P. Yeh, "Optical waves in layered media", Wiley Series in Pure and Applied Optics, (1988). 